\title{
3 Konzeptionen von Bildungsgerechtigkeit und deren Bearbeitung in der Organisation Schule
}

Sollen Orientierungen der Förderung und Selektion in Schulen in Zusammenhang mit deren Anforderungen und Funktionen untersucht werden, ist es erforderlich, einen näheren Blick auf verschiedene Konzeptionen von Bildungsgerechtigkeit in der Organisation Schule zu werfen. In einem ersten Schritt wird der Begriff der Bildungsgerechtigkeit von verschiedenen Perspektiven der Chancenungleichheit abgegrenzt. Es schließt sich eine Betrachtung verschiedener Konzeptionen von Bildungsgerechtigkeit als sensibilisierendes Konzept der Arbeit an. Dies schafft die Grundlage dafür, im folgenden Kapitel 4 die Sinnherstellung in der Organisation Schule im Hinblick auf die Bearbeitung von Bildungsgerechtigkeit hin zu kontextualisieren. Die Reflexion der verschiedenen Akteursperspektiven zielt darauf ab, die Rolle der Organisation in der Herausbildung von handlungsleitenden Orientierungen zu Förderung und Selektion analysieren zu können.

\subsection{Abgrenzung der Bildungsgerechtigkeit vom Begriff der Chancenungleichheit im Bildungssystem}

In den letzten Jahren wird in der erziehungswissenschaftlichen Literatur vermehrt der Begriff der Bildungsgerechtigkeit anstelle des Begriffs der Chancenungleichheit im Bildungssystem verwendet. Giesinger (2015, S. 151) sieht diese Entwicklung in $\mathrm{Zu}$ sammenhang mit der Priorität des Begriffs der equity in der englischen Forschung. Daneben fällt auf, dass ebenfalls in internationalen Vergleichsstudien wie PISA mit dem Begriff der Chancengerechtigkeit gearbeitet wird (Niedegger et al., 2014). Unabhängig von dieser Dominanz des Begriffs der Bildungsgerechtigkeit im aktuellen bildungspolitischen und wissenschaftlichen Diskurs soll im Anschluss analysiert werden, inwiefern sich die Begrifflichkeiten und die damit verbundenen theoretischen Konzeptionen hinsichtlich des impliziten Verständnisses von Individuen und Bildung unterscheiden. $\mathrm{Um} \mathrm{zu}$ argumentieren, weshalb in dieser Arbeit mit verschiedenen Konzepten der Bildungsgerechtigkeit gearbeitet wird, wird in einem ersten Schritt die wissenschaftliche und bildungspolitische Verwendung verschiedener Chancenungleichheitskonzepte analysiert. In einem zweiten Schritt wird dann von diesen Verständnissen von Chancengleichheit abgegrenzt.

Mit Blick auf die Bearbeitung von Chancenungleichheit im Bildungssystem wird in wissenschaftlichen Studien insbesondere zwischen einer strukturtheoretischen und einer entscheidungstheoretischen Perspektive unterschieden (vgl. u. a. Kronig, 2007). 
Beide Perspektiven verfolgen gemäß Emmerich und Hormel (2013, S. 138) allokationstheoretische Überlegungen und lenken die Aufmerksamkeit von den Ursachen ungleicher Bildungsbeteiligungen auf die einzelnen Individuen und deren Leistungskapazitäten. Eigenrationalitäten des Bildungssystems oder auch der Schule als Organisation werden hingegen nicht fokussiert (Emmerich \& Hormel, 2013). Die Perspektiven gehen von unterschiedlichen Vorstellungen von Individuen und deren Kapazität zu rationalen Entscheiden aus. Adressieren sie bei der Frage nach den Ursachen bestehender Chancenungleichheiten im Bildungssystem verschiedene Ebenen, werden organisational eingeschriebene Chancenungleichheiten nicht thematisiert. Aus diesem Grund werden die beiden Perspektiven um eine dritte, nämlich eine organisationssoziologische Perspektive ergänzt. Neben der Systemebene (strukturtheoretische Perspektive) und der individuellen Ebene (entscheidungstheoretische Perspektive) kommt so eine institutionelle/organisationale Ebene hinsichtlich der Analyse von Chancenungleichheiten im Bildungssystem in den Fokus. Im Anschluss wird jeweils ein kurzer theoretischer Abriss dieser drei Perspektiven dargestellt. Daneben folgt eine Auseinandersetzung mit den damit verbundenen Annahmen und Implikationen. Im Anschluss an die drei Unterkapitel wird ein Fazit der Verwendungspotentiale und Limitationen gezogen.

\subsubsection{Strukturtheoretische Perspektive}

Die Forderung, Chancenungleichheit aufgrund erblich erworbener Merkmale durch das Bildungssystem zu verringern, geht gemäß Böttcher (2002) mit der Auflösung der ständischen Ordnung zu Beginn des 19. Jahrhunderts sowie der Institutionalisierung des Schulwesens einher. Dabei ist in der Kritik der schichtspezifischen Verteilung von Bildungstiteln die Prämisse enthalten, dass Begabung und Talent in der Gesellschaft prinzipiell gleichmäßig verteilt sind (Kronig, 2007). Diese Prämisse wird durch eine „,apologetische Begabungsideologie“ (Böttcher, 2002, S. 38) gebrochen, die auf Blindheit gegenüber sozialen Ursachen von Bildungschancen beruht und von privilegierten Schichten im Bildungssystem verteidigt wird. Bourdieu und Passeron (1971) beschreiben diese Ideologie folgendermaßen:

„Die privilegierten Klassen rechtfertigen ihre kulturellen Privilegien mit einer Ideologie, die man als charismatisch bezeichnen könnte (da sie nach „Gaben“ und „Begabung“ wertet). Wobei die Gesellschaft in individuelle Begabung oder persönlichen Verdienst umgewertet wird. (...) Diese Alchemie gelingt umso besser, als die unteren Klassen ihr keineswegs ein anderes Bild des Studienerfolges entgegenstellen, sondern ihrerseits den Essentialismus der oberen Klassen übernehmen und ihre Unterprivilegierung als persönliches Schicksal erleben“ (Bourdieu \& Passeron, 1971, S. 85).

Bourdieu und Passeron (1971) kritisieren die Begabungsideologie an französischen Eliteschulen, welche von der oberen Klasse entworfen wird, jedoch nur deshalb funk- 
tioniert, weil sich die unteren Klassen ihrem ,Schicksal' ergeben. Diese Ideologie diene dazu, soziale Ungleichheiten zu verschleiern und Sozialstrukturen zu verfestigen. Den Bildungsinstitutionen wird hier unterstellt, sich nur sehr langsam zu transformieren und durch einen eigenständigen Habitus auf Organisationsebene die Strukturen der Gesellschaft langfristig zu perpetuieren (Bourdieu \& Passeron, 1971, S. 212). In ihrem Werk „Die Illusion der Chancengleichheit“ untersuchen die Autoren mit dieser theoretischen Perspektive die Perpetuierung der Gesellschaftsordnung anhand unterschiedlicher Zugangschancen zu Hochschulbildung nach sozialer Klasse. Anhand von Daten der französischen Hochschulstatistik aus dem Jahre 1961/62 zeigen Bourdieu \& Passeron (1971), dass die untersten Klassen verschwindend geringe Chancen für einen Hochschulbesuch haben, während diese bei Kinder von Führungskadern ,achtzigmal größer" sei (ebd., S. 20).

Der strukturtheoretische Erklärungsansatz der Reproduktion von Chancenungleichheiten durch gesellschaftliche Institutionen wurde in den Erziehungswissenschaften der 1970er Jahren eher spärlich aufgenommen und erlebte erst Mitte der 1980er Jahre, insbesondere in der Diskussion des viel beachteten Werks ,Die feinen Unterschiede، (Bourdieu, 1982), eine verbreitete Anerkennung (Liebau, 2006). In diesem wird eine gegliederte Gesellschaft auf der Basis sozialer Felder skizziert, wobei den Individuen aufgrund unterschiedlicher schichtspezifischer Möglichkeitsräume Positionen zugewiesen sind (Bourdieu, 1982, S. 188). In Abgrenzung zu zweckrationalen Handlungsmodellen werden Ungleichheitsstrukturen als vorab definierte Lebensstile im sozialen Raum gedeutet, welche das Handlungswissen der Akteure strukturieren (ebd., S. 729). Bildungsinstitutionen werden dabei als Teil der Distributionsinstanzen in der schichtspezifischen Reproduktion sozialer Ungleichheiten angesehen (ebd., S. 147). Hinsichtlich der schulischen Akteure werden unter dieser Perspektive insbesondere der Habitus von Lehrpersonen und (fehlende) Passungsverhältnisse zum Habitus verschiedener Schüler/-innengruppen diskutiert (vgl. Kapitel 4.1). Der strukturtheoretische Ansatz fokussiert verborgene Mechanismen der Reproduktion sozialer Verhältnisse. Hinsichtlich Bildung fokussiert die Perspektive eingeschriebene Reproduktionsmechanismen gesellschaftlicher Institutionen. Diese struktur- und kulturtheoretischen Überlegungen von Bourdieu wurden gegen Ende der 1980er Jahre in der deutschen erziehungswissenschaftlichen Forschung häufig und teilweise auch kritisch reflektiert (Kramer, 2011). Die Kritik bezog sich dabei insbesondere auf verschiedene Aspekte einer deterministischen Vorstellung sich selbst reproduzierender Institutionen, welche gesellschaftlichen Wandel kaum erklären lassen (ebd., 2011).

Während das Thema der Chancenungleichheit in den 1990er Jahren weniger im erziehungswissenschaftlichen Fokus stand, wurde mit dem Theoriemodell von Bourdieu wieder häufiger in den 2000er Jahren gearbeitet, einhergehend mit der bildungspolitischen Diskussion als Reaktion auf internationale Leistungsmessungsstudien wie PISA. Diese empirischen Rezeptionen der 2000er Jahre bezeichnet Kramer (2011, S. 31) als „Muster der bruchstückhaften, verkürzten Rezeption“. Als eine solche ver- 
kürzte Rezeption können beispielsweise die in der PISA-Studie aufgenommenen Struktur- und Prozessmerkmale familiärer Herkunft gezählt werden, welche sich am EGP-Klassenmodell nach Erikson, Goldthrope und Portocarero (1979) orientieren (vgl. Baumert, 2006; Prenzel, Saelzer, Klieme \& Koeller, 2013; Watermann \& Baumert, 2006). Dabei wird eine verkürzte Darstellung des Bourdieu'schen Ansatzes in einer reduzierten empirischen Operationalisierung von Habitus und des Vorhandenseins verschiedener Formen von Kapital übertragen (Kramer \& Helsper, 2010).

Aktuelle Bezugnahmen auf den strukturtheoretischen Ansatz bezeichnet Kramer als „würdigende wie produktiv erweiternde Rezeption“ (2011, S. 31). Solche Erweiterungen können beispielsweise in Arbeiten gesehen werden, in welchen das transformative Potential der Bourdieu'schen Theorie (vgl. Mills, 2008) oder auch HabitusTransformationen von Schüler/-innen diskutiert werden (Kramer, Helsper, Thiersch \& Ziems, 2013). Im Unterschied dazu gehen Studien zum Habitus von Lehrpersonen von relativ stabilen Konzeptionen und Denkgewohnheiten hinsichtlich der beruflichen Maxime aus (vgl. u. a. Reusser \& Pauli, 2014). Die Autoren bezeichnen unterbewusste kollektive Praktiken von berufstypischen Wahrnehmungen und Bearbeitungsformen als „berufsbezogene Überzeugungen“" von Lehrpersonen (Reusser \& Pauli, 2014, S. 645-646).

Die Kritik am strukturtheoretischen Ansatz wird insbesondere in seinem gesellschaftlichen Determinismus sowie der fehlenden Wahrnehmung der Akteure begründet (vgl. u. a. Kronig, 2007, S. 66-67). Bourdieu selbst betont insbesondere in seinen frühen Schriften die Stabilität des Habitus und im Hinblick auf Institutionen des Bildungssystems deren perpetuierende Wirkung auf gesellschaftliche Strukturen (Bourdieu \& Passeron, 1971). Auch wenn Überlegungen zu Transformationsprozessen oder zum Habituserwerb in neueren Rezeptionen vorhanden sind, bietet sich das Konzept kaum dafür an, eine Perspektive des Wandels gesellschaftlicher Wertvorstellungen im Bildungssystem einzunehmen. In empirischen Studien werden daneben die zentralen Konzepte Habitus und Kapital häufig in einer verkürzten Weise operationalisiert, was auf die Schwierigkeit einer sinnvollen Operationalisierungsmöglichkeit solcher latenter Strukturen hinweist. Neben diesen theoretischen und empirischen Limitationen ermöglicht der Erklärungsansatz von Bourdieu zwar eine systemkritische Perspektive auf Chancenungleichheiten im Bildungssystem, schulische Akteure werden jedoch insbesondere als Vertreter (und Vollzieher) der Institution Schule verstanden. Chancenungleichheit bedeutet, nimmt man die empirische Studie von Bourdieu \& Passeron als Beispiel, die ungleiche Verteilung von Zugangschancen basierend auf Herkunftsunterschieden. Es wird somit auf gleiche Chancen am Ziel (Ende der obligatorischen Schule) unabhängig von der sozialen Herkunftsschicht abgezielt und somit eine meritokratische Logik auf Bildung vertreten. Möchte man jedoch 1) Akteure im Bildungssystem und deren Konzeptionen untersuchen und 2) alternative Bildungsideale neben einer meritokratischen Logik miteinbeziehen, eignet sich der Ansatz kaum als theoretische Grundlage. 


\subsubsection{Entscheidungstheoretische Perspektive}

Während die strukturtheoretische Perspektive von beschränkten Handlungsmöglichkeiten von Individuen auf der Grundlage von in Strukturen eingeschriebenen Ungleichheiten fokussiert, untersucht der entscheidungstheoretische Ansatz gerade die Handlungsmöglichkeiten und Spielräume von Individuen. Dabei wird das Individuum als Vertreter einer Gruppe verstanden. Entsprechend untersucht die Perspektive herkunftstypisches Entscheidungshandeln verschiedener Akteursgruppen.

Im entscheidungstheoretischen Diskurs dominieren in der aktuellen Diskussion insbesondere Studien, welche den Einfluss des Elternhauses auf gruppenspezifische Leistungsunterschiede und auf das Entscheidungshandeln an Übergängen im Bildungssystem untersuchen. So finden sich im Anschluss an die theoretischen Arbeiten zur Entstehung von Bildungschancen zahlreiche Studien zur Erforschung von primären und sekundären Herkunftseffekten (Boudon, 1974). Dieser zu den Rational-ChoiceTheorien zählende Ansatz rückt die Entstehung und Perpetuierung von Bildungsungleichheiten aufgrund von schichtspezifisch variierenden Bildungsentscheidungen in den Mittelpunkt. Dabei wird zwischen primären und sekundären Herkunftseffekten unterschieden. Primäre Effekte beschreiben die schulische Performanz aufgrund schichtspezifischer Erziehung, Ausstattung und Förderung, während unter sekundären Effekten schichtspezifische elterliche Bildungsentscheidungen insbesondere an Übergängen gefasst werden (Boudon, 1974, S. 29-30).

Empirische Arbeiten, welche mit diesem Theoriemodell arbeiten, gibt es sowohl in Deutschland (u. a. Becker \& Lauterbach, 2010; Blossfeld, 2013; Ditton, 2010; Relikowski, Schneider \& Blossfeld, 2010) als auch in der Schweiz (u. a. Biewer, Wandeler \& Baeriswyl, 2013; Kost, 2013; Maaz, Baeriswyl \& Trautwein, 2011; Stadelmann-Steffen, 2013; Wolter, 2013) in großer Zahl. Für Deutschland analysieren beispielsweise Becker und Lauterbach (2010) basierend auf dem Modell der Herkunftseffekte die Sozialisation im Elternhaus als wichtige Ursache für Bildungsungleichheit, wobei die Schule ungleiche Startbedingungen kaum auszugleichen vermag (Becker \& Lauterbach, 2010). Weiter zeigen Studien für Deutschland und die Schweiz eine Persistenz sozial ungleicher Bildungschancen von Schülerinnen und Schülern aufgrund der Zugehörigkeit zu einer gewissen Schicht oder Migrationsgruppe (Maaz, 2006; Stadelmann-Steffen, 2013). Biewer et al. (2013) untersuchen im Rahmen einer Vollerhebung der Primarschul-Sekundarschul-Übertrittjahrgänge 2009/2010 des deutschsprachigen Teils des Kantons Freiburg $(\mathrm{CH})$ primäre und sekundäre Herkunftseffekte sowie Gerechtigkeitswahrnehmungen von Eltern im Übertrittverfahren. Die Autoren kommen zu dem Schluss, dass sowohl seitens der Lehrperson als auch seitens der Eltern statusabhängige Zuweisungsempfehlungen im Sinne von sekundären Herkunftseffekten auftreten (Biewer et al., 2013, S. 438).

Wie soeben aufgezeigt, fokussieren sich erziehungswissenschaftliche Untersuchungen mit einer entscheidungstheoretischen Perspektive insbesondere auf Chancen- 
ungleichheiten im Bildungssystem basierend auf Herkunftseffekten. Individuen und ihre Merkmale als Quelle der Ungleichheitsreproduktion rücken im Unterschied zum strukturtheoretischen Erklärungsansatz in den Fokus (Hirschauer, 2014, S. 172). Das Handeln von Akteuren wird dabei einerseits als rational angesehen und andererseits als schichtspezifisch geprägt. Dieser Ansatz wird insofern kritisiert, als dass rationales Wahlhandeln und damit vollständig informierte Individuen vorausgesetzt werden (Kronig, 2007). Daneben zeigt sich ein blinder Fleck der Analysen darin, dass Leistung als Herkunftsmerkmal charakterisiert und damit die organisationale Beteiligung an der individuellen Zuschreibung von Leistung nicht reflektiert wird (Emmerich \& Hormel, 2013). Dieser Zugang bleibt dem Individuum und dessen Entscheidungsverantwortung verhaftet, womit, wie Böttcher (2002) kritisiert, der Erfolg im Bildungssystem einzig an der Leistungsfähigkeit des Individuums festgemacht wird, während gesellschaftliche und institutionelle Strukturen eine Blackbox bleiben.

In einer entscheidungstheoretischen Perspektive werden die Nutzer von Bildungssystemen als entscheidungsrational handelnde Individuen unterschiedlicher Herkunft angesehen. Bildungsungleichheiten lassen sich aufgrund solcher Entscheidungsrationale der Nutzer erklären, welche durch die Akteure auf der Angebotsseite verstärkt werden. Die Funktion von Bildung wird insbesondere auf seine Selektionsfunktion hin reduziert, wobei Leistungsoutputs der Bildung (abgebildet im ersten Herkunftseffekt) sowie an den Übergängen im Bildungssystem (zweiter Herkunftseffekt) im Zentrum stehen. Weitere Funktionen von Bildung, die nicht auf Selektion ausgerichtet sind, sind hingegen nicht im Fokus dieses theoretischen Paradigmas. Daneben hat eine Perspektive auf Individuen, welche ihr entscheidungsrationales Handeln gerade erst durch Bildungsprozesse ausbilden können, kaum einen Platz in dieser Perspektive.

\subsubsection{Organisationstheoretische Perspektive}

Eine weitere Perspektive auf die Ursachen von Chancenungleichheiten im Bildungssystem findet sich in organisationstheoretischen Ansätzen. Im Unterschied zum strukturtheoretischen und zum entscheidungstheoretischen Erklärungsansatz werden in dieser Perspektive die Entstehung und Reproduktion von Chancenungleichheiten im Bildungssystem nicht an schulexternen Faktoren festgemacht, sondern in eingeschriebenen Diskriminierungsformen schulischer Organisationen (Hormel, 2010).

Einen überindividuellen Erklärungsansatz für die Reproduktion von Chancenungleichheiten durch schulische Organisationen bietet beispielsweise das Konzept der institutionellen Diskriminierung. Diskriminierende Praktiken gründen ihm zufolge nicht im Handeln der Akteure, sondern sind formalen Regeln, etablierten Strukturen, gesellschaftlichen Wertvorstellungen und Gewohnheiten institutionalisierter Organisationen eingeschrieben (Gomolla \& Radtke, 2009). Entscheidungsprämissen basieren auf organisationalen Routinen und sind dabei selbstreferentiell; sie ergeben sich in der 
Aushandlung zwischen Umwelterwartungen sowie professionellen und organisationalen Entscheidungen (Hormel 2010, S. 177-178). Ungleiche Bildungsbeteiligungen und Schulerfolge werden gemäß diesem Erklärungsansatz durch unhinterfragte Wahrnehmungsmuster, Normen und Routinen schulischer Organisationen stabilisiert (Gomolla $\&$ Radtke, 2009). Soziales Handeln wird in dieser Perspektive von im Voraus definierten, sozial gültigen Deutungsmustern in Institutionen für handelnde Subjekte ermöglicht und begrenzt (Bommes \& Radtke, 1993, S. 489). Empirische Studien, welche Ungleichheiten im Bildungssystem aus organisationsperspektivischer Sichtweise untersuchen, basieren häufig auf nicht standardisierten Daten. So werden Differenzierungspraktiken an Schulen in einer organisationstheoretischen Perspektive insbesondere mit rekonstruktiven Ansätzen analysiert (Fölker \& Hertel, 2015; Gomolla, 2005; Maier, 2016; Sturm \& Wagner-Willi, 2016). Während Gomolla (2005) anhand exemplarischer Fallstudien im Ländervergleich (Ö, CH, E) Praktiken institutioneller Diskriminierung betrachtet, untersuchen Sturm und Wagner-Willi (2016) Differenzkonstruktionen von Lehrpersonen anhand der Kategorie Leistung im Unterricht. Weiter rekonstruieren Maier (2016) sowie Fölker und Hertel (2015) kollektive Orientierungen im Hinblick auf Selektion und Differenzierung. Während Maier am Beispiel von Notenkonferenzen organisationssoziologischen Befunden von Begründungsmustern bei gleichzeitiger Aufrechterhaltung der professionellen Fassade nachgeht, rekonstruieren Fölker und Hertel (2015) schulspezifische Formen pädagogischer Orientierungen an zwei segregierten Großstadtschulen in Deutschland.

Eine organisationstheoretische Perspektive auf Ungleichheitsproduktion findet sich daneben im Ansatz des doing difference von West und Fenstermaker (1995a, zit. nach Kubisch, 2008, S. 19-20). Das aus der Genderforschung (unter dem Begriff doing gender) stammende Forschungsparadigma betont den aktiven Anteil an Unterschiedsproduktionen durch Organisationen. Der Ansatz des doing difference ist einem konstruktivistischen Forschungsparadigma zuzuordnen, insofern er davon ausgeht, dass soziale Differenzierung kommunikativ hergestellt wird (Hirschauer, 2014, S. 173). Dabei wird argumentiert, dass neben dem Arbeitsmarkt Organisationen wie die Schule maßgeblich an der (Re-)Produktion von Bildungsungleichheiten beteiligt sind.

Als Mehrwert dieser Ansätze zu der entscheidungstheoretischen und strukturtheoretischen Perspektive wird der Fokus auf die Schule selbst und eingeschriebene Chancenungleichheiten im Bildungssystem gelegt. Dabei wird durch den Diskriminierungsbegriff das Ergebnis vorweggenommen, das Bestehen und die Reproduktion von Chancenungleichheiten scheinen systemimmanent. Akteure werden als Kollektive verstanden, schulinterne Aushandlungsprozesse kommen hingegen nicht in den Blick. 


\section{Kombination}

In verschiedenen Studien werden Erklärungsansätze kombiniert, um eine mehrperspektivische Betrachtung auf die Entstehung und den Umgang mit Chancengleichheiten im Bildungssystem zu erhalten. Kronig (2007) kombiniert strukturtheoretische und entscheidungstheoretische Erklärungsansätze, um empirische Evidenzen für die ,systematische Zufälligkeit des Bildungserfolges' im Schweizer Bildungssystems nachzuzeichnen. Im kantonalen Vergleich von Trackingsystemen betrachten Felouzis und Charmillot (2013) aus einer systemtheoretischen und organisationstheoretischen Perspektive die Organisation des Übergangs zu nachobligatorischen Bildungsstufen. Insbesondere der Organisation des "trackings“ und weniger der heterogenen oder homogenen Form des segregierten Systems wird ein ungleichheitsreproduzierender Charakter zugesprochen (Felouzis \& Charmillot, 2013, S. 200). Während die strukturelle Einbettung als relevant angesehen wird, geschieht die Organisation der Differenzierung auf der Ebene schulischer Einzelinstitutionen. Auch diesen Kombinationen verschiedener Erklärungsansätze der Ursache von Chancenungleichheiten im Bildungssystem ist gemein, dass grundsätzlich von einem meritokratischen Bildungsideal ausgegangen wird.

\subsubsection{Fazit und Abgrenzung}

Die verschiedenen Perspektiven auf die Entstehung und Bearbeitung von Chancenungleichheiten im Bildungssystem ermöglichen, insbesondere in deren kombinierten Betrachtung, Ursachen ungleicher Chancen verschiedener Schüler/-innengruppen zu verschiedenen Zeitpunkten im Bildungssystem zu ergründen. Während Eigenrationalitäten der Schule als Organisation in einer strukturtheoretischen und entscheidungstheoretischen Perspektive kaum fokussiert werden, kommen diese in einer organisationstheoretischen Betrachtung in den Blick (Emmerich \& Hormel, 2013). Organisationstheoretische und strukturtheoretische Erklärungsansätze fokussieren hingegen nicht das Handeln einzelner Akteure, welche sowohl auf der Nutzer- als auch auf der Anbieter-Seite als Kollektive verstanden werden. Den Ansätzen gemeinsam ist der Blick auf das Individuum des Schülers (und teilweise auch der Schulakteure) als Vertreter seiner Herkunftsfamilie und die Schule als Ort, an welchem Chancenungleichheiten bearbeitet oder reproduziert werden. Hinsichtlich Bildung unterliegen die Ansätze einem grundsätzlich meritokratischen Bildungsideal. Dieses Bildungsideal orientiert sich an Leistung als gerechtem Gradmesser von Selektionsprozessen. Insofern wird Chancenungleichheit letztendlich an der Nichterreichung hoher Leistungsziele für gewisse Gruppen festgemacht. Alternative Funktionen von Bildung, welche sich weniger an diesem Leistungsoutput orientieren, kommen kaum in den Blick. Alle diese theoretischen Perspektiven, welche unter dem Chancenungleichheitsbegriff ausgearbeitet 
wurden, fragen danach, wie ein meritokratisches Leistungsideal im Bildungssystem erreicht werden könnte respektive aus welchen Gründen dieses Ideal nicht erreicht wird. Eine alternative Betrachtung im Sinne humanistischer Bildungsideale der allgemeinen Menschenbildung (vgl. Kapitel 2.1) scheint jedoch im Rahmen dieser Perspektiven nicht denkbar. In der dominanten empirischen Operationalisierung von ungleichheitsrelevanten Faktoren zeigt sich weiter eine Reduktion insbesondere auf Leistungsoutputs verschiedener Schüler/-innengruppen zu einem bestimmten Zeitpunkt. Fähigkeiten von Individuen werden dabei auf messbare schulische Leistungsoutputs reduziert, während andere Fähigkeiten (im Sinne Nussbaums, 2014) kaum thematisiert werden. Diese empirische Verkürzung, ebenso wie die theoretischen Angebote, die sich um den Begriff der Chancenungleichheit im Bildungssystem entwickelt haben, führen zur Herausforderung, dass die Konnotation des Begriffes mit der Erreichung von Leistungsoutputs verknüpft wird. Die Theorieangebote bleiben somit bei der Frage der gerechten Verteilung von Noten, Übertrittchancen und Bildungstitel verhaftet. Der Chancenungleichheitsdiskurs in der Erziehungswissenschaft ist dabei Outputgerichtet. Das Konzept eignet sich insbesondere für Studien, welche am Leistungsoutput gemessene Chancenungleichheiten im Bildungssystem für verschiedene Gruppen von Schülerinnen und Schülern untersuchen möchten. Dies kann in einer kombinierten Sichtweise verschiedener Entstehungs- und Prozessursachen je nach Forschungsstand differenziert beurteilt werden. Gerade für die Schulakteure kann jedoch eine solch Output-orientierte Sichtweise problematisch sein, welche sich kaum mit pädagogischen Idealen ganzheitlicher Bildung vertragen (vgl. dazu Heinrich, 2013). Der Begriff der Chancenungleichheit eignet sich daher wenig dazu, normative Konstruktionen verschiedener Schulakteure zu analysieren und welchen Verständnissen von Bildung und Individuen diesen zugrunde liegen.

Zur Beantwortung dieser Fragen nach verschiedenen Verständnissen der Bildung von Individuen eignet sich hingegen der Bildungsgerechtigkeitsbegriff. Während, wie im Anschluss diskutiert, es auch hier theoretische Konzeptionen von Chancengerechtigkeit im Bildungssystem gibt, welche eine meritokratische Leistungslogik vertreten, lassen sich ebenfalls alternative Theorieangebote finden. Bildungsgerechtigkeit lässt als Begriff ebenfalls zu, nicht (nur) ausgehend vom Output zu diskutieren, sondern ebenfalls nach Schwellenkonzepten, Prozessen und der Auseinandersetzung mit gesellschaftlichen Wertvorstellungen zu fragen. Bildungsgerechtigkeit kann unterschiedlich verstanden werden, woraus sich für das Bildungssystem und für die individuelle Bildung verschiedene Zielsetzungen ergeben. Mit dem Begriff der Bildungsgerechtigkeit anstelle der Chancenungleichheit ist eine solch ergebnisoffene Diskussion möglich, gerade auch in Auseinandersetzung und Abgrenzung von dominanten gesellschaftlichen Vorstellungen der Bildungsgerechtigkeit. Die Frage nach unterschiedlichen Konzeptionen von Bildungsgerechtigkeit verschiedener Schulakteure und Schulkollektive steht im Zentrum dieser Arbeit. Im Anschluss werden theoretische Konzeptionen von Bildungsgerechtigkeit diskutiert, welche die bildungspolitischen und wissen- 
schaftlichen Diskussionen prägen. Ausgehend von der Annahme, dass Bildungsgerechtigkeit ein konzeptspezifisches und möglicherweise auch ein schulkollektives Konstrukt darstellt, gilt es dann, verschiedene Konzeptionen von Bildungsgerechtigkeit im Hauptteil dieser Arbeit empirisch zu rekonstruieren.

\subsection{Konzeptionen von Bildungsgerechtigkeit: Verteilung, Anerkennung oder Befähigung?}

In diesem Kapitel soll der Frage nach den Zielsetzungen verschiedener Bildungsgerechtigkeitskonzeptionen auf bildungspolitischer Ebene, aber auch auf Organisations- und Akteursebene nachgegangen werden. Giesinger (2007, S. 367) spricht davon, dass mit dem Bildungsgerechtigkeitsansatz ein Schwellenkonzept angedacht werden kann. An welcher Stelle diese Schwelle der gerechten Verteilung von Bildungsgütern ansetzt, ist eine Frage gesellschaftlicher Aushandlung und der Zielsetzung von Bildungsprozessen. Der Vorteil des Schwellenkonzepts sieht Giesinger darin, dass es nicht grundsätzlich als ungerecht angesehen werden muss, wenn verschiedene Personen $\mathrm{zu}$ einem bestimmten Zeitpunkt nicht dasselbe Leistungsniveau erreichen (Giesinger, 2007, S. 379). Eine Herausforderung ergibt sich dadurch, dass in bildungspolitischen Debatten sowohl der Begriff der Bildung als auch der Begriff der Gerechtigkeit als etwas, das es anzustreben gilt, häufig unscharf verwendet werden (Dietrich et al., 2013).

Der Bildungsgerechtigkeitsbegriff erlebte im bildungspolitischen Diskurs im Nachgang an internationale Vergleichsstudien wie PISA eine neue Popularität, während in den 1980er und 1990er Jahren der Fokus noch stärker auf der inneren Reformierung der Schule lag. In dieser durchaus positiv zu wertenden Popularisierung der Diskussion wurden die Begriffe und das Verständnis von Bildung und Gerechtigkeit selbst jedoch wenig reflektiert. Anstelle von Bildung rückt der Potentialbegriff der Schüler/-innen in den Fokus, wodurch die Leistungsfähigkeit im Zentrum des Diskurses steht (Thieme, 2013, S. 163). Bildungsgerechtigkeit wird dabei verstanden als „Chancengleichheit im Sinne einer Startchancengleichheit, die der Logik der Responsibilisierung folgt und mit einem funktionalistisch verkürzten Verständnis von Bildung einhergeht" (Thieme, 2013, S. 164, Hervorhebungen im Original). Responsibilisierung meint die Eigenverantwortung für das Ergebnis von Bildungsleistungen, sobald eine Startchancengleichheit hergestellt ist. Heinrich (2013, S. 129) spricht in diesem Zusammenhang von Anstrengungsbereitschaft, welche das Ziel einer auf Leistungsgerechtigkeit abzielenden Konzeption darstellt.

Diesen Output-Fokus des Chancengleichheitsdiskurses reflektieren Dietrich, Heinrich und Thieme (2013) in ihrem Werk Bildungsgerechtigkeit jenseits von Chancengleichheit kritisch. Sie sehen im Begriff der Chancengleichheit eine Verkürzung von Bildungsgerechtigkeitskonzeptionen, welche die bildungspolitische Diskussion im 
Anschluss an die PISA-Debatte dominiert. Die Verkürzung zeigt sich gemäß Heinrich (2015, S. 241) darin, dass eine Orientierung an einem an Konkurrenz festgemachten Prinzip den Ansprüchen einer Bildungsgerechtigkeitsnorm nicht nachzukommen vermag, da in diesem Prinzip auch bei einem fairen Kampf um die knappe Ressource Bildung Ungleichheit als Ergebnis unweigerlich angelegt ist. Gleichheit wirft im Unterschied zu Gerechtigkeit immer eine Verteilungsfrage auf. Die Vorstellung von Chancengleichheit beinhaltet implizite Annahmen von Gerechtigkeit und Bildung, welche jedoch weder expliziert noch reflektiert werden (Dietrich, Heinrich \& Thieme, 2013, S. 17). So wird Bildungserfolg oder der alternativ verwendete Begriff der Kompetenz insbesondere Output-orientiert verstanden und mit der Erhöhung der gesellschaftlichen Humankapitalproduktion verknüpft (vgl. u. a. Bänninger, 2016; Otto \& Schrödter, 2010).

Es ist schwierig, eine für das Bildungswesen sinnvolle Definition des Gerechtigkeitsbegriffes zu finden. Einerseits kann der Begriff kaum, wie beispielsweise in der Wirtschaft oder auf dem Arbeitsmarkt, als gleiche Belohnung für gleiche Leistung gedacht werden, da Bildungsleistungen auch von personalen Faktoren der Kinder und ihrer Eltern abhängen. Andererseits sollten in einem weiteren Bildungsverständnis Aspekte wie Autonomie und Verantwortungsübernahme für Bildungsleistungen mitgedacht werden (vgl. Stojanov, 2011, S. 16). Wird Bildungsgerechtigkeit insbesondere über gerechte Startchancen definiert, geraten der weitere Prozess der Bildung und dessen systemische Bedingungen aus dem Blick. Das Erreichen von Leistungszielen wird dann als individuelle Leistung oder individuelles Scheitern betrachtet. Dieses wenig prozessorientierte Verständnis zeigt sich beispielsweise in der Formulierung des meritokratischen Ideals, welches auf Leistungsgerechtigkeit bei gleichen Startbedingungen, jedoch individuellen Verläufen und Entscheiden ausgerichtet ist (vgl. u. a. Eckert \& Gniewosz, 2017; Stojanov, 2011). Der Bildungsgerechtigkeitsbegriff wird darüber hinaus in der bildungspolitischen Diskussion oftmals mit zweckrationalen Zielen verknüpft, insbesondere mit dem besseren Abschneiden in internationalen Leistungstests (Stojanov, 2011, S. 27).

Es bestehen also, wie diese Hinführung zeigt, weder für das Verständnis von Gerechtigkeit noch für Bildungsprozesse als Vollzugsmechanismus einheitliche Konzeptionen. Bildungspolitische und wissenschaftliche Debatten beziehen sich vielmehr auf verschiedene Bildungsgerechtigkeitskonzeptionen, meist ohne dieses Verständnis explizit offenzulegen. In bildungspolitischen Debatten findet häufig das Konzept der distributiven Verteilungsgerechtigkeit Anwendung. Als Alternativen werden insbesondere Befähigungs- oder Anerkennungsgerechtigkeitskonzeptionen formuliert (vgl. u. a. Bänninger, 2016; Dietrich et al., 2013; Nussbaum, 2014; Stojanov, 2011). Es ist davon auszugehen, dass diese Diskurse in der pädagogischen Praxis wahrgenommen und rezipiert werden. Die unterschiedlichen Verständnisse von Bildungsgerechtigkeit werden daher als Grundlage für die empirische Rekonstruktion verschiedener Gerechtigkeitsvorstellungen in der pädagogischen Praxis im Folgenden einzeln betrachtet. 


\subsubsection{Verteilungsgerechtigkeit}

Ein wichtiger Bezugspunkt für den Ansatz der Verteilungsgerechtigkeit ist John Rawls' Standardwerk A Theory of Justice (1971). Als zentrale Grundprinzipien einer gerechten Gesellschaft formuliert Rawls (1971, S. 61) einerseits das Prinzip gleicher Grundfreiheiten (größtmögliche individuelle Freiheit, begrenzt durch die der anderen Personen) und andererseits ein Differenz- oder Fairnessprinzip (bestehende soziale und wirtschaftliche Ungleichheiten sind gerecht zu gestalten). Dabei definiert er eine Liste von Grundfreiheiten (,primary social goods“). Die beiden Prinzipien sind hierarchisch zu denken: Das Differenzprinzip ist dem Freiheitsprinzip untergeordnet, gilt aber nur dann als erfüllt, wenn die Ungleichheit von allen Parteien als gerecht wahrgenommen wird (ebd., S. 62). Rawls' Gerechtigkeitskonzeption basiert insbesondere auf dem Konzept der Fairness, das der Autor im Artikel „Justice as Fairness“ wie folgt definiert: „fundamental of justice, is the concept of fairness which relates to right dealing between persons who are cooperating with or competing against one another $[\ldots]]^{\text {“ }}$ (Rawls, 1958, S. 178). In dieser Definition wird das liberale Konkurrenzprinzip als Gesellschaftsvertrag nicht hinterfragt; es geht um gleiche Ausgangsbedingungen oder, in den Worten von Rawls (1971, S. 83), um "fair equality of opportunity“. An die Begrifflichkeit der „equal opportunities“ und der individuellen Verantwortung für rationelle Entscheidungen knüpft Arneson (1989) an. So sieht er gleiche Möglichkeiten ungleicher Entscheidungsrationale als sinnvolle Basis einer Verteilungsgerechtigkeitskonzeption (Arneson, 1989). In Abgrenzung von einer Vorstellung der gleichen Verteilung von Ressourcen - wozu gemäß Arneson auch Talente zu zählen sind - gilt eine gleiche Verteilung der Möglichkeiten, diese zu erreichen, als gerecht (Arneson, 1989, S. 89).

An einer gerechten Verteilung orientierte Ziele können verschiedene Prinzipien verfolgen. Verteilungsgerechtigkeit kann auf formale/rechtliche oder relationale Gleichheit abzielen, sie kann aber auch nach Verdiensten respektive Bedürfnissen fragen. Der Fokus in Bildungsdebatten liegt vorwiegend auf den beiden Letztgenannten (Bänninger, 2016, S. 101). Relationale Gleichheit ist laut Hübner (2013, S. 44) weniger als Kriterium für das Bildungssystem geeignet, da eine Angleichung nach unten in Nachteilen für privilegierte Gruppen ausschlagen würde. Vielmehr wird darüber diskutiert, wie Bedürfnisgleichheit oder eher Verfahrensgleichheit im Bildungssystem erreicht werden kann. Gemäß Hübner (2013, S. 47) werden als Zielsetzung oftmals Kombinationen verschiedener Formen von Verteilungsgerechtigkeit formuliert, wobei das gesellschaftliche Begründungsverfahren im Zentrum steht. Ein attraktiver Begründungsansatz zeichnet sich dadurch aus, dass Güterverteilungen so legitimiert werden können, dass sich ein intelligentes Individuum dafür entscheiden würde, gleichzeitig die Prozedur jedoch möglichst so weit verhüllt ist, dass aufgrund fehlender Informationen eine unparteiliche Entscheidung gefällt wird (ebd., S. 51-52). 
Im Bildungssystem ist gemäß Hübner (2013) neben dieser kollektiven Moral auch eine individuelle Moral gefordert, was uns zum dominierenden bildungspolitischen Gerechtigkeitsdiskurs führt. In ihm wird insbesondere eine Vorstellung von Gerechtigkeit als gerechte Verteilung von Startchancen vertreten, wobei die Schüler/-innen im Anschluss selbst für Leistungserfolge verantwortlich sind (Stojanov, 2011, S. 28). Diese Idee orientiert sich an der meritokratischen Vorstellung, dass bei gleichen Startbedingungen das Individuum seine Leistungsmöglichkeiten gemäß seinem individuellen kognitiven Potential mehr oder weniger ausschöpfen kann. Die Bestimmung kognitiver Ziele orientiert sich dabei an standardisierten Entwicklungsverläufen, wobei soziale Konstruktionsprozesse von Leistungsnormen außer Acht gelassen werden (Bänninger, 2016). Verteilungsgerechtigkeit wird als „Begabungsgerechtigkeit" (Stojanov, 2013, S. 58) verstanden, Begabungen werden jedoch zumeist als biologisch-genetisches Potential gesehen, das bereits vor Eintritt in das Bildungssystem besteht. Laut Thieme (2013, S. 164) geht mit dieser Lesart des Potentialbegriffs das Verständnis eines Bildungsprozesses einher, dem zufolge den Schülerinnen und Schülern gleiche Startchancen geboten werden sollen, um ihre Potentiale zu entwickeln, während die Verwirklichung an sie delegiert wird.

Das Ziel der Verteilungsgerechtigkeit bezieht sich auf Theorien distributiver Gerechtigkeit. In der dominanten Verwendung wird es insbesondere auf das Kernstück der Kompensation von Herkunftsungleichheiten reduziert (Stojanov, 2011, S. 29). Diese in der Bildungspolitik häufig vertretene Konzeption von Gerechtigkeit durch Bildung zielt somit auf den Ausgleich von Ungleichheiten durch die Primärsozialisation bei formeller Gleichbehandlung aller Schüler/-innen nach ihren Leistungen (Stojanov, 2011, S. 28). Insofern dominiert die Logik der Untersuchung ungerechtigkeitsproduzierender Ursachen von Bildungswegen insbesondere anhand individueller Merkmale der Schüler/-innen, Eltern und Lehrpersonen. Wenig reflektiert wird dabei die Rolle von Institutionen, Organisationen und Akteuren, die sie für die Chancengerechtigkeit nicht nur zu Beginn, sondern auch im weiteren Prozess der Schulzeit und bei Vergabe von Bildungszertifikaten spielen. Es lässt sich in weiten Teilen des vorherrschenden bildungspolitischen Diskurses eine Individualisierung der Verantwortung in einer an distributiver Herkunftsgerechtigkeit orientierten Sichtweise festmachen. Soziale Konstruktionen von Gerechtigkeit werden dabei ebenso oft ausgeblendet wie ein prozessuales Verständnis sich wandelnder Begabungspotentiale im Rahmen der jeweiligen Möglichkeiten. Indem sie von natürlichen Begabungsunterschieden ausgehen, entlasten sich Bildungsinstitutionen und legitimieren so die Reproduktion sozialer Ungleichheiten. Dies aber führt die Diskussion um die Reproduktion von Chancenungerechtigkeit ad absurdum (Stojanov, 2013, S. 60). Aus der Perspektive der Schulakteure lässt sich zudem argumentieren, dass eine Bildungsgerechtigkeitskonzeption, die lediglich an Eigenverantwortung und Anstrengungsbereitschaft der Schüler/-innen appelliert, einem humanistischen pädagogischen Verständnis von Bildung, das auf Beziehung ausgerichtet ist, entgegensteht (Heinrich, 2013, S. 186-187). 
Als Errungenschaft einer liberalen, auf Prinzipien der Verteilungsgerechtigkeit basierenden Bildungsgerechtigkeitskonzeption kann allerdings die hohe Bekanntheit und weitgehende Anerkennung des Strebens nach Gerechtigkeit im Bildungssystem angesehen werden. Dies erst ermöglicht es, sich kritisch mit dem Konzept von Bildungsgerechtigkeit auseinanderzusetzen. Diese Bekanntheit bringt jedoch kritischen Stimmen zufolge die Gefahr der unreflektierten (und teilweise verkürzten) Übernahme gesellschaftspolitischer Ziele in das Bildungssystem mit sich. So grenzt sich beispielsweise Honneth von Rawls' liberaler Gerechtigkeitskonzeption ab, sieht er doch in der liberalen Konzeption eine Verkürzung von Bildungsgerechtigkeit, da nur die Sicherung subjektiver Freiheitsrechte und die gerechte Verteilung von Gütern thematisiert wird, nicht aber die soziale Bedürftigkeit von Anerkennungsbeziehungen (Honneth, 2013). Martha Nussbaum und Amartya Sen (1993) verweisen auf den idealtypischen Charakter in Rawls' Gerechtigkeitstheorie, welche das Verhalten von Individuen zu wenig berücksichtige, und halten dem einen Capability Approach entgegen. Beide alternativen Konzeptionen von Bildungsgerechtigkeit werden im Anschluss mit Blick auf ihre Relevanz für die Erziehungswissenschaft und die pädagogische Praxis differenziert betrachtet.

\subsubsection{Anerkennungsgerechtigkeit}

Während der Begriff der Verteilungsgerechtigkeit die distributive Verteilung insbesondere sozioökonomischer Güter fokussiert und auf Individuen abzielt, adressiert die Anerkennungsgerechtigkeit symbolische Teilhabe wie Autonomie und Mitsprache (Bänninger, 2016). Soziale Konstruktionen von Bildungsgerechtigkeit durch Akteure, aber auch durch Institutionen werden anders als bei Verteilungsgerechtigkeitsansätzen mit berücksichtigt. Der anerkennungstheoretische Diskurs in der deutschsprachigen Erziehungswissenschaft bezieht sich einerseits auf die Schriften von Axel Honneth, mit denen er sich von liberalen Gerechtigkeitstheorien abgrenzt (Honneth, 2010). Andererseits geht er auf die reformpädagogische Perspektive der „Pädagogik der Vielfalt“ von Annelore Prengel (1993-2013) zurück, die sich an den menschenrechtlichen Prinzipien Gleichheit, Freiheit und Solidarität orientiert (Prengel, 2013). Balzer und Ricken (2010) wie auch Stojanov (2011) knüpfen an diese und weitere Diskurslinien an und verfeinern den Begriff der Anerkennung.

Anerkennung ist sowohl im alltagsweltlichen Diskurs als auch im wissenschaftlichen Verständnis eng mit Wertschätzung und Respekt verknüpft. In Bezug auf die sozialphilosophischen Arbeiten Honneths ist sie als moralethische Kategorie zu fassen (Balzer \& Ricken, 2010). So formuliert Honneth (2010) drei gesellschaftliche anerkennungstheoretische Ideale als Bedingung für die individuelle und soziale Freiheit: Liebe/Empathie, moralischer Respekt und soziale Wertschätzung. Prengel (2013, S. 60, 90) verortet diese Begriffe im Kontext pädagogischer Beziehungen in der Soli- 
darität mit Fremden (Liebe), der Achtung altersgerechter gleicher Freiheit (Respekt) und einer kindgerechten Form der leistungsbezogenen Wertschätzung. Individuen werden als in gesellschaftliche Institutionen eingebettet angesehen, in denen sie auf die Anerkennung anderer angewiesen und nur so in der Lage sind, sich als vollwertige Mitglieder der Gesellschaft zu verstehen (Honneth, 2013, S. 294). Gerecht sind Veränderungen in der Gesellschaft dann, wenn mehr Personen als zuvor befähigt werden, eine in ihren Augen gehaltvolle Identität zu entwickeln (ebd., S. 294). In einer ähnlichen Lesart formuliert Prengel pädagogische Beziehungen als lebensgeschichtlich bedeutsam. Bei einem Gelingen dieser Beziehungen pendeln sie sich zwischen den Polen Anerkennung, Verletzung und ambivalentem Verhalten ein (Prengel, 2013, S. 11).

Stojanov (2013) arbeitet den Anerkennungsgerechtigkeitsansatz nach Honneth weiter aus. Zentral für die Empathie vor allem im frühen Kindheitsalter sind die Bezugspersonen, die Bedürfnisse und Wünsche des Individuums zurückspiegeln. Respekt wird als überindividuelle Eigenschaft angesehen, jedes Individuum mit Würde und der Fähigkeit zu autonomem Handeln auszustatten. Soziale Wertschätzung ist in dieser Sicht die Synthese der individualisierten Empathie und des universalen Respekts, bei dem die Fähigkeitspotentiale des Einzelnen gesellschaftlich anerkannt werden (ebd., S. 64). Gerechtigkeit im Bildungswesen zeichnet sich demnach dadurch aus, dass diese drei Formen von Anerkennung - Empathie, Respekt und soziale Wertschätzung - als Orientierungsmaßstab für pädagogisches Handeln gelten. Eine Bildungsgerechtigkeitsvorstellung, die auf einer leistungsbasierten Verteilung von Berufsmöglichkeiten und Lernressourcen nach Begabung oder erbrachten Leistung basiert, wird hingegen zurückgewiesen. Als Vorstellung von Bildungsgerechtigkeit formuliert Stojanov:

„Bildung ist an sich kein Gut, das man besitzt, sondern sie bezeichnet letztlich den Prozess der Entwicklung individueller Autonomie, welche die Voraussetzung für Subjektivität, für eine aktive und selbstbestimmte Lebensführung ist. Bildungsinstitutionen sollen diesen Entwicklungsprozess nach Kräften bei jedem einzelnen Kind und Heranwachsenden fördern, ohne sich dabei anzumaßen, seine spätere berufliche und soziale Stellung vorzubestimmen“"(Stojanov, 2013, S. 62).

In diesem Zitat sind zentrale Annahmen des Konzepts der Anerkennungsgerechtigkeit enthalten. In Abgrenzung vom Ansatz der Verteilungsgerechtigkeit wird Bildung nicht als ,Gut' oder ,Besitz' verstanden. Vielmehr steht die Entwicklung individueller Autonomie eines jeden einzelnen Kindes im Zentrum. Zentrale Aufgabe von Bildungsinstitutionen ist dabei die Schaffung sinnvoller Voraussetzungen zur Erreichung dieser Autonomie, wobei der Qualität von Sozialbeziehungen eine zentrale Stellung zukommt (vgl. Stojanov, 2011, S. 40). Für Bildungsinstitutionen setzt dies voraus, dass Bedingungen geschaffen werden können, in welchen Schüler/-innen die Möglichkeit haben, ihre Identität zu entwickeln. Ein gerechtes Bildungssystem zeichnet sich dadurch aus, dass es keine als statisch angenommene Begabung und Kognition zuordnet, sondern variable Kompetenzpotentiale anerkennt (Stojanov, 2013, S. 64). 
Obwohl grundsätzlich kaum bestritten werden dürfte, dass Anerkennung die Entwicklung von Fähigkeiten begünstigt und eine anhaltende Nichtanerkennung der Entwicklung schaden kann, wird die positivistische Normativität des Begriffs kritisiert (Balzer, 2014; Balzer \& Ricken, 2010; Giesinger, 2013). Wird Anerkennung wie bei Honneth oder auch Stojanov als moralische Prämisse verstanden, lässt sich dies laut Balzer und Ricken (2013, S. 53-55) nur begrenzt mit der pädagogischen Praxis vereinen. Das enge Verständnis von Anerkennung auf „nur positive, wertschätzende und wohlwollende Handlungen“" (Balzer \& Ricken, 2010, S. 53) verdrängt weitere Handlungsgründe und erlaubt es nicht mehr, die Möglichkeiten zur Erreichung von Anerkennung in Frage zu stellen. Zudem werden die in das pädagogische Handeln eingeschriebenen Widersprüche und Machtverhältnisse ausgeblendet (ebd., 2010, S. 54-55). Balzer (2014, S. 19-22) fordert demgegenüber, Anerkennung auf ihren Machtcharakter hin zu befragen und dem praktischen Vollzug von Anerkennung in pädagogischen Beziehungen nachzugehen. Kritisiert wird die Normativität des Ansatzes, welche Anerkennung vorwiegend positiv konnotiert und Abweichungen ignoriert (Balzer, 2014). Unter Verweis auf Judith Butler (2009) heben Balzer und Ricken (2010, S. 67) hervor, dass Anerkennung nicht nur eine Bestätigung von Subjekten sein kann, sondern auch einen hierarchischen Charakter hat, insofern die Anerkennung des Anderen immer mit einer Bestätigung des Eigenen einhergeht. Im pädagogischen Kontext wollen Balzer und Ricken (2010, S. 70) Anerkennung daher ambivalent als Bestätigung/Negierung, Ermöglichung/Einschränkung sowie Unterstützung/Disziplinierung verstanden wissen. Die Ambivalenz pädagogischer Beziehungen hebt auch Prengel (2013) hervor und verweist auf das Machtverhältnis in Generationenbeziehungen, welches aus Ambivalenzen relationaler Beziehungen der Anerkennung und Beschämung hervorgeht (Prengel, 2013, S. 34). Im schulischen Alltag werden, wie die Beobachtungsstudie INTAKT zeigt, auch verletzende Handlungsmuster als Teil pädagogischen Handelns akzeptiert, was Prengel (2013, S. 128) auf die Tabuisierung in öffentlichen Debatten zurückführt.

Basierend auf dieser Kritik ließe sich in Anlehnung an Balzer und Ricken (2010) ein breiterer Begriff der Anerkennung in Zusammenhang mit der pädagogischen Adressierung sowohl als Wahrnehmung einzelner Individuen als auch als Differenzierung und Zuweisungspraktik verstehen. Laut Prengel (2013) kommt pädagogisches Handeln kaum in einer reinen Form als anerkennend oder beschämend vor, sondern ist von Ambivalenzen geprägt. Dieses Verständnis schützt vor einer normativen Wertung (Anerkennung als Ziel einer (reform-)pädagogischen Moral) und lässt Raum für weiterführende Interpretationen hinsichtlich der Bearbeitung von Bildungsgerechtigkeit. Anerkennung ist so im pädagogischen Kontext nicht nur die positive Wertschätzung der Individualität einzelner Schüler/-innen, sondern im gleichen Zuge ein In-Beziehung-Setzen des Individuums mit gesellschaftlichen und schulischen Normalitätserwartungen. Anerkennungsgerechtigkeit kann in diesem Verständnis ergebnisoffen gelesen werden. 


\subsubsection{Befähigungsgerechtigkeit oder Capability Approach}

Im Unterschied zum Verteilungsgerechtigkeitsansatz geht der Befähigungsgerechtigkeitsansatz von einer ganzheitlichen Perspektive auf das Individuum aus. Er grenzt sich von der distributiven Ausprägung der laut John Rawls (1971) anzustrebenden ,Grundgüter' ab. Vermieden werden soll gemäß Nussbaum (2014, S. 34-35) ${ }^{5}$ einerseits die Konzeption einer ,schwachen Theorie“ des Guten (zugunsten einer ,starken Theorie' des Guten), andererseits die Reduktion insbesondere auf Einkommensungleichheiten sowie die Akzeptanz von Ungleichheiten, wenn sich dadurch die Position aller in der Gesellschaft verbessert. Die Konzeption der gerechten Verteilung von Gütern berücksichtigt aus dieser Sicht das einzelne Individuum und dessen unterschiedlichen Bedarf an Gütern zu wenig (Nussbaum, 2014, S. 36). Dieser unterschiedliche Bedarf betrifft Eigenschaften (wobei im Bereich der Bildung der Mehranspruch auf finanzielle Unterstützung für Kinder aus benachteiligten Minderheitsgruppen als Beispiel genannt wird) ebenso wie die Fähigkeit, überhaupt Entscheide treffen zu können (ebd., S. 36-39). Diese Fähigkeit wird im Unterschied zu einer liberalen Konzeption nicht einfach als vorhanden, sondern als von unterschiedlichen Gewohnheiten und Lebensweisen geformt angesehen, welche in der Formulierung eines ,guten Lebens' mitgedacht werden müssen (ebd., S. 40).

Diese Kritik am Ansatz der Verteilungsgerechtigkeit mündet somit in einer ,Capability“-Perspektive (Nussbaum \& Sen, 1993; Sen, 1992). In ihr geht es um die Frage nach der Befähigung aller Personen zum Erlangen eines guten Lebens. Wurde der Ansatz vom indischen Ökonomen Amartya Sen bereits ab 1979 entwickelt, ist für die vorliegende Untersuchung die weitere Ausarbeitung durch die Philosophin und Genderforscherin Martha Nussbaum geeignet, da sie im Unterschied zu Sen eine konkrete Ausgestaltung von notwendigen Befähigungen respektive Grundfähigkeiten formuliert. Die reine Teilhabe an begehrten Grundgütern wird in einer Befähigungsgerechtigkeitsperspektive als nicht ausreichend angesehen. Dispositionen, Vermögen und Möglichkeiten von Individuen sollten ebenso in die Analyse einbezogen werden (Schrödter, 2013). Der Fokus auf konkrete Tätigkeiten ermöglicht gemäß Nussbaum (2014, S. 38) aufzuzeigen, welche von Individuen angestrebten Ziele zu Spannungen und Konflikten in der Gesellschaft führen können. Individuen verfügen dabei über verschiedene personale Kompetenzen, die sie unter sich ändernden Kontextbedingungen befähigen, Ressourcen unterschiedlich zu nutzen (Schrödter, 2013, S. 78-81). Im Unterschied zum Anerkennungsgerechtigkeitsansatz werden ungleiche Dispositionen der Individuen berücksichtigt. Fähigkeiten oder Kompetenzen sind nicht einfach individuell gegeben, sondern hängen von sozialen Kontextbedingungen ab, welche das Individuum befähigen oder behindern. Hinsichtlich des Bildungssystems fordert Schrödter

5 Deutsche Ausgabe des Werks The qualitiy of life, 8. Auflage, herausgegeben von Herlinde Pauer-Studer. 
(2013, S. 84) aus diesem Grund, keine Gleichverteilung von Mitteln anzustreben, da dies aufgrund der Ungleichheit der Individuen zu struktureller Diskriminierung führe. Ähnlich wie Rawls' (1971) Grundfreiheiten formuliert Nussbaum eine Liste minimaler Grundfähigkeiten zur Erreichung eines menschenwürdigen Lebens. Jenseits einer rein distributiv gedachten Verteilungsgerechtigkeit werden weitere partizipative Fähigkeiten wie Emotionen, soziale Beziehungen, Verbundenheit mit der Natur, Spiel als Voraussetzungen eines guten Lebens etc. formuliert (Nussbaum, 2014, S. 57-58). Die Liste enthält nicht nur Fähigkeiten, welche der einzelnen Person dienen, sondern auch solche der sozialen Teilhabe. Beispielsweise werden die „Fähigkeit, sich eine Vorstellung vom Guten zu machen und kritisch über die eigene Lebensplanung" zu sein, oder die „Fähigkeit, für andere und bezogen auf andere zu leben“, als Grundfähigkeiten formuliert (Nussbaum, 2014, S. 58). Diese Befähigungen versteht Nussbaum im Unterschied zu Sen als grundsätzliche verfassungsmäßige Rechte, für deren Erreichung Bildungsinstitutionen eine wichtige Rolle spielen. So fordert sie auch mit Blick auf das Bildungswesen einen ganzheitlichen Ansatz. Es reiche nicht aus, sich auf Tätigkeiten wie die Bereitstellung von Ressourcen für die Bildung zu beschränken:

„Er [der Ansatz] meint nicht, die Bereitstellung von Ressourcen für die Erziehung sei ausreichend - insbesondere dort nicht wo Menschen in widrigen Umständen leben -, sondern er entwirft phantasievolle Programme, die es benachteiligten Gruppen ermöglichen, die Ressourcen wirklich zu ihrem Vorteil zu nutzen“(Nussbaum, 2014, S. 71).

Freiheit bzw. Entscheidungsfreiheit werden in dieser Logik als Vermögen definiert, sich für oder gegen die Realisierung der Ausbildung der eigenen Fähigkeiten zu entscheiden.

Indem so festgelegt wird, welche Möglichkeiten und Fähigkeiten anzustreben sind, wird das philosophische Konzept empirisch mess- und rekonstruierbar. Allerdings besteht die Gefahr einer Reduktion auf die formulierten Fähigkeiten. Dem Auflisten von Grundbefähigungen, welche Otto und Ziegler (2008, S. 12) angesichts ihrer empirischen Überprüfbarkeit als Stärke des Ansatzes von Nussbaum sehen, begegnet Sen (1992) mit Zurückhaltung. Er fordert, eine globale Festlegung zu vermeiden (zit. nach Steckmann, 2008). Die Kritik am Capability Approach bezieht sich denn auch gerade auf die Frage nach dem Verständnis eines „erstrebenswerten Lebens“, auf die objektive Bestimmung, was gut und erstrebenswert ist. Allerdings teilt der Ansatz laut Otto und Ziegler (2008, S. 10-11) eine solche Ideologie ,objektiv' erstrebenswerten Lebens gerade nicht, sondern stellt die Entscheidungsfreiheit des Individuums ins Zentrum. Kritisiert wird dennoch die paternalistische Prägung des Ansatzes, welcher „bevormundende Eingriffe in individuelle Entscheidungsräume“ legitimiere (Steckmann, 2008, S. 107). Dieser Verdacht des Paternalismus wird jedoch teilweise dadurch entkräftet, dass nicht eine Konzeption des erstrebenswerten Lebens dargeboten wird, sondern Individuen dazu befähigt werden sollen, ihren eigenen Lebensentwurf zu erstellen (Steckmann, 2008). Eine weitere Gefahr könnte im Hinblick auf die Bereitstel- 
lung von besonderen Unterstützungs- und Lernangeboten im Bildungssystem darin bestehen, dass durch die Sichtbarmachung bestehender Unterschiede diese reproduziert werden.

\subsection{Gerechtigkeitskonzeptionen im Bildungsdiskurs}

Gemeinsam ist den diskutierten Gerechtigkeitskonzeptionen das Ziel, Unterschiede in demokratischen Gesellschaften und auch im Bildungssystem aufzudecken, welche mit einer demokratischen Vorstellung von Bildungsgerechtigkeit konfligieren. Bildungsinstitutionen kommt allen drei Konzeptionen zufolge eine zentrale Stellung für die Integration von Individuen in die Gesellschaft zu. Allerdings liegen ihnen unterschiedliche Verständnisse der Aufgabe von Bildungsinstitutionen sowie des Individuums zugrunde. Grundsätzlich kann unterschieden werden zwischen einer liberalen, an individueller Leistungsfähigkeit und Entscheidung orientierten Logik und einer humanistischen Logik der Bildungsgerechtigkeit, die auf eine ganzheitliche Förderung des Individuums ausgerichtet ist und eine non-distributive Konzeption von Bildungsgerechtigkeit vertritt. Die liberale Verteilungsgerechtigkeitskonzeption zielt auf Verfahrens- und Prozessgerechtigkeit ab oder, wie dies Rawls (1971) formuliert, auf ,equal opportunities‘. Das Bildungssystem soll, basierend auf gleichen Startbedingungen, eine faire Distribution begehrter Ressourcen wie Bildungstitel ermöglichen. Die Verteilungerechtigkeitsvorstellung basiert auf dem Konzept der Fairness zwischen kompetitiven und kooperierenden Individuen. Während also ungleiche Startbedingungen im Bildungssystem durch zusätzliche Ressourcen ausgeglichen werden sollen, werden die Entscheidungskompetenzen der Individuen nicht hinterfragt. Leistung wird als Kognition oder Talent verstanden, die unabhängig von der sozialen Einbettung unterschiedlich ausgeprägt sind und durch Bildungsprozesse entsprechend gefördert werden sollen. Systemische oder organisationale Reproduktionsmechanismen und weitreichende Selektionsentscheide werden als ,faire' Marktbedingungen angesehen.

In Abgrenzung dazu wird im Anerkennungs- wie auch im Befähigungsgerechtigkeitsansatz ein humanistisches Bildungsverständnis vertreten. Beide Konzepte sehen es als Aufgabe des Bildungssystems an, Kinder zu entscheidungsfähigen Mitgliedern der Gesellschaft auszubilden, bevor weitreichende Entscheidungen in gerechter Weise möglich sind. Dabei steht nicht die gerechte Distribution (gleicher) Ressourcen im Zentrum, sondern vielmehr die Frage nach der Teilhabe und Mitsprache eines jeden Individuum im Sinne eines Empowerments. Die beiden Ansätze unterscheiden sich darin, inwiefern unterschiedliche Bedürfnisse und Voraussetzungen von Individuen für eine Bildungsgerechtigkeitskonzeption mitgedacht werden. Während der Anerkennungsgerechtigkeitsansatz auf die Entwicklung individueller Kapazitäten wie Autonomie und Subjektivität ausgerichtet ist und dabei bewusst bestehende Unterschiede ausklammert, versucht der Befähigungsgerechtigkeitsansatz diese Unterschiede zu 
fokussieren und an den Dispositionen und Möglichkeiten von Individuen anzusetzen. Was das Bildungssystem betrifft, könnte man vorsichtig formulieren, dass Anerkennung eher im Sinne des ursprünglichen Inklusionsgedankens einer ,Bildung für alle“ zu verstehen ist. Der Capability Approach setzt im Sinne Nussbaums hingegen an den Systembedingungen an und kritisiert diese, wenn sie nicht darauf abzielen, Personen mit ihren unterschiedlichen Voraussetzungen dahingehend zu bilden und zu fördern, dass sie dieselben Möglichkeiten und Fähigkeiten der Entscheidungsfindung haben.

In der Literatur finden sich einige Hinweise für die Übernahme verschiedener Gerechtigkeitskonzeptionen im bildungspolitischen und pädagogischen Diskurs. In bildungspolitischen Diskussionen und wissenschaftlichen Studien im Nachgang der Ergebnisse internationaler Leistungstests wird häufig eine (verkürzte) Verteilungsgerechtigkeitskonzeption vertreten (vgl. u. a. Bänninger, 2016; Stojanov, 2013; Honneth, 2013). Den Schülerinnen und Schülern wird, sind gerechte Grundbedingungen geschaffen, die alleinige Verantwortung für ihre Leistungserfolge zugewiesen. In der pädagogischen Praxis besteht dann aber die Gefahr, dass diese Verteilungsgerechtigkeitslogik vom pädagogischen Ethos des professionellen Einzelfallbezugs abweicht (Heinrich, 2013, S. 168). Eine liberale Bildungsgerechtigkeitsvorstellung, die auf Eigenverantwortung basiert, kann gemäß dem Autor auf der Seite der Lehrperson zu einem Konflikt zwischen gesellschaftlichen Bildungsgerechtigkeitsvorstellungen und subjektiven pädagogischen Werten führen. Im Extremfall erleben sich die Pädagogen in ihrem Berufsauftrag nicht mehr als handlungsfähig.

Im Anschluss an den PISA-Diskurs wird die Bildungsgerechtigkeitsdebatte insbesondere auch mit Blick auf die Einführung inklusiver Bildungssysteme geführt. Grundsätzlich ist die Forderung nach Inklusion an einer Gerechtigkeitsvorstellung im Sinne der Anerkennung - Bildung für alle - orientiert. Inklusion ist in seiner ursprünglichen Begrifflichkeit gemäß Heinrich (2015, S. 252) in einer ganzheitlichen, humanistischen Vorstellung von „Bildung zur Seite des Subjekts“ zu verstehen. Da Inklusionsbestrebungen jedoch auch politische Folge des schlechten Abschneidens in internationalen Leistungstests sind, wurde die Debatte nicht nur im Sinne der Teilhabe an Bildungsinhalten, sondern auch mit dem Ziel steigender Schulleistungen aller Schüler/-innen geführt. So folgen gemäß Bänninger (2016, S. 99) aktuelle bildungspolitische Diskurse mehrheitlich der neoliberalen Vorstellung, Schüler/-innen bereits früh zu eigenständigen Subjekten ,im Sinne eines unternehmerischen Selbst“ auszubilden und ihnen die Verantwortung für ihren Leistungserfolg zu übertragen. Der Anspruch der Leistungssteigerung und Leistungsnormorientierung stellt Inklusion als Teilhabe an gleichen Bildungszielen vor eine schwer zu bewältigende Herausforderung. Gerade hinsichtlich der Einführung inklusiver Bildungssysteme zeigt sich in den bildungspolitischen Diskussionen ein Spannungsfeld verschiedener Bildungsgerechtigkeitsvorstellungen (Sturm, Köpfer \& Wagener, 2016).

Zusammenfassend kann davon ausgegangen werden, dass im bildungspolitischen Diskurs ebenso wie in der Output-orientierten Schuleffektivitätsforschung eine an Ver- 
teilungsgerechtigkeit orientierte Bildungsgerechtigkeitskonzeption dominiert. In der Begründung von Reformbemühungen integrativer und inklusiver Beschulung zeigen sich daneben auch noch Diskurse eines Anerkennungsgerechtigkeitsansatzes. Wird Bildung hingegen als Befähigung verstanden mit dem Ziel, Individuen mit ihren unterschiedlichen Bedürfnissen zu entscheidungsfähigen Mitgliedern der Gesellschaft zu machen, und werden Systembedingungen, welche dies verhindern, kritisiert, so deutet dies auf ein Verständnis im Sinne der Befähigungsgerechtigkeit hin. Bei der empirischen Rekonstruktion von Bildungsgerechtigkeitskonzeptionen, welche sich in Schulen insbesondere in der Organisation von Förderung und Selektion zeigen, kann es als zentral angesehen werden, diese unterschiedlichen Konzeptionen von Bildungsgerechtigkeit im pädagogischen Kontext zu reflektieren. Dabei wird eine konstruktivistische Perspektive eingenommen in der Annahme, dass Konzeptionen von Bildungsgerechtigkeit auf diskursiv hergestellten Konstruktionsprozessen von Individuen, Gruppen und Organisationen basieren. Inwiefern sich diese verschiedenen Konzeptionen von Bildungsgerechtigkeit in schulischen Organisationen rekonstruieren lassen, gilt es empirisch zu prüfen. Dabei wird davon ausgegangen, dass Bildungsgerechtigkeitskonzeptionen nicht nur auf bildungspolitischer oder individueller Ebene, sondern auch auf der intermediären Ebene bestehen. Es stellt sich damit die Frage, inwiefern die Schule als Organisation dazu beitragen kann, kollektive Gerechtigkeitskonzeptionen zu generieren. Solche Sinnherstellungsprozesse in schulischen Organisationen werden im folgenden Kapitel besprochen.

Open Access Dieses Kapitel wird unter der Creative Commons Namensnennung 4.0 International Lizenz (http://creativecommons.org/licenses/by/4.0/deed.de) veröffentlicht, welche die Nutzung, Vervielfältigung, Bearbeitung, Verbreitung und Wiedergabe in jeglichem Medium und Format erlaubt, sofern Sie den/die ursprünglichen Autor(en) und die Quelle ordnungsgemäß nennen, einen Link zur Creative Commons Lizenz beifügen und angeben, ob Änderungen vorgenommen wurden. Die in diesem Kapitel enthaltenen Bilder und sonstiges Drittmaterial unterliegen ebenfalls der genannten Creative Commons Lizenz, sofern sich aus der Abbildungslegende nichts anderes ergibt. Sofern das betreffende Material nicht unter der genannten Creative Commons Lizenz steht und die betreffende Handlung nicht nach gesetzlichen Vorschriften erlaubt ist, ist für die oben aufgeführten Weiterverwendungen des Materials die Einwilligung des jeweiligen Rechteinhabers einzuholen.

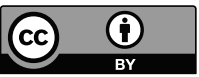

